\title{
Determining the Fetal Inflammatory Response in an Experimental Model of Intrauterine Inflammation in Rats
}

\author{
MICHAEL J. BELL, JOHN M. HALLENBECK, AND VITTORIO GALLO \\ Center for Neuroscience Research, Children's Research Institute [M.J.B., V.G.], Children's National Medical \\ Center, George Washington University School of Medicine, Washington, D.C., 20010, and Stroke Branch \\ [M.J.B., J.M.H.], National Institute of Neurological Diseases and Stroke, Bethesda, Maryland, 20892
}

\begin{abstract}
ABS
Intrauterine infection is a risk factor for developmental brain
injuries in childhood. A variety of cytokines known to be toxic to
developing brain cells have been isolated from mothers or chil-
dren at risk for developmental disabilities, and these cytokines
have been proposed as mediators of these injuries. We have
developed a model of intrauterine inflammation that damages the
developing white matter and we now hypothesize that selected
cytokines are increased after our experimental inflammatory
stimulus. Timed-pregnant Fischer 344 and Lewis rats were in-
jected with 0.1 mg/kg of lipopolysaccharide (LPS) into the cervix
at E15. Tumor necrosis factor- $\alpha$ (TNF- $\alpha$ ), interferon- $\gamma$ (IFN- $\gamma$ ),
IL-6, and IL-10 were measured in homogenates of fetal brain and
placenta at serial time periods within the first $24 \mathrm{~h}$ after the
inflammatory stimulus. TNF- $\alpha$ was increased 20 -fold in the
placenta and more than 5-fold in the fetal brain after the stimulus.
IFN- $\gamma$ was only increased within the fetal brain (20-fold) and
IL-6 was only increased in the placenta (10-fold). IL-10 was
mildly increased in the placenta and was decreased slightly in the
fetal brain. Our observations show that an intrauterine inflam-
\end{abstract}
Inflammation has become a more widely recognized risk factor for the development of brain injuries in children (1-4). Over the past several years, epidemiologic evidence has accumulated implicating inflammation and infection with $\mathrm{CP}$ and PVL. Determining the mechanisms responsible for this association is pivotal in developing strategies to ameliorate the injuries in an early stage or preventing them altogether.

Cytokines are soluble mediators of the immune system that are produced in response to infections and also play a role in

Received December 2, 2003; accepted April 4, 2004

Correspondence: Michael J. Bell, M.D., Center for Neuroscience Research, Children's Research Institute, Department of Critical Care Medicine, Children's National Medical Center, 111 Michigan Ave., NW, Washington, DC 20010; e-mail: mbell@cnmc.org

Supported by an Intergovernmental Personnel Agreement between the National Institute of Neurological Disorders and Stroke (NINDS) and George Washington University School of Medicine, K12 HD01399, and K08 HD044716 with National Institute of Child Health and Human Development (NICHD).

DOI: 10.1203/01.PDR.0000139407.89883.6B matory stimulus can cause large increases in Th1 cytokines within the fetal brain. The placenta can produce selected cytokines but fails to produce IFN- $\gamma$, suggesting that the fetal immune system produces this cytokine in response to our stimulus. By studying placental and brain cytokine responses in models such as ours, the mechanisms responsible for the damage to developing white matter can be determined. (Pediatr Res 56: 541-546, 2004)

$\quad$ Abbreviations
CNP, 2', 3'-cyclic nucleotide phosphodiesterase
CP, cerebral palsy
IFN- $\boldsymbol{\gamma}$, interferon gamma
LPS, lipopolysaccharide
PLP, proteolipid protein
PVL, periventricular leukomalacia
TLR4, toll-like receptor 4
TNF- $\boldsymbol{\alpha}$, tumor necrosis factor alpha

normal brain development (5). Th1 cytokines promote the immune response to infection, whereas Th2 cytokines quell the inflammatory response. TNF- $\alpha$ is a Th1 cytokine that can be detected in developing rodent astrocytes during fetal life (6) and plays a role in parturition initiation in the placenta $(7,8)$. In response to inflammatory stimuli such as LPS, TNF- $\alpha$ is rapidly released by microglia as well as other cells within the CNS $(9,10)$. TNF- $\alpha$ is implicated in many forms of degenerative brain injuries and can initiate programmed cell death signaling cascades. IFN- $\gamma$ is a Th1 cytokine that is not normally present within the CNS during development. IFN- $\gamma$ is released from Th1 lymphocytes and natural killer cells and it can induce the release of TNF- $\alpha$ from a variety of cell types $(11,12)$. After an inflammatory stimulus, IFN- $\gamma$ activates microglia, converts astrocytes into antigen presenting cells, and up-regulates major histocompatibility type II antigens on neurons, astrocytes, and oligodendrocytes (13-17). IL-6 is another Th1 cytokine that is produced by macrophages and monocytes 
in the systemic circulation and it promotes immunoglobulin secretion and $\mathrm{T}$ cell activation (18). Its role in normal CNS development is unclear, but IL-6 increases rapidly in response to LPS and TNF- $\alpha$ (19). IL-6 receptors are present on both neurons and glia (20) and it has been implicated in both protective (neurotrophin, cellular survival) as well as degenerative processes within the CNS (21). IL-10 is a Th2 cytokine that acts to limit the inflammatory response after stimulus. Its role in normal development is controversial, but IL-10 diminishes the inflammatory actions of Th1 cytokines through a wide variety of actions (22).

Th1 cytokines have been implicated in inflammationinduced developmental brain injuries, but Th2 cytokines have not. TNF- $\alpha$ and IL- 6 have been isolated from the placenta, amniotic fluid, and umbilical venous blood, and IFN- $\gamma$ has been isolated from fetal blood in children that develop $\mathrm{CP}$ or PVL (2,23-26). TNF- $\alpha$ immunoreactivity has been demonstrated in PVL lesions at autopsy (27). In vitro systems have demonstrated that TNF- $\alpha$ decreases oligodendrocyte progenitor number, affects cell morphology, and inhibits maturation, whereas IFN- $\gamma$ induces apoptosis [as measured by deoxynucleotidyl transferase-mediated dUTP nick-end labeling (TUNEL) and annexin V binding] and decreases oligodendrocyte proliferation $(28-30)$. These observations have led to hypotheses that these cytokines produced in response to intrauterine infection and inflammation cause damage to the developing fetal brain. Conversely, although Th2 cytokines can be produced by placental tissues (31) and have been associated with protection from certain brain injuries $(32,33)$, there is no evidence to date that they play a role in inflammation-induced developmental brain injuries (34).

We have developed a model of intrauterine inflammation in timed-pregnant rats to study this process (35). Our model shows i) damage to the developing white matter as demonstrated by decreased oligodendrocyte-specific staining (CNP and PLP) and ii) an increase in apoptotic cell death in the periventricular region as manifested by an increased number of TUNEL-stained nuclei in the periventricular region. We concluded that these observations could be the result of a decreased number of oligodendrocytes, a decreased amount of myelin protein produced by the oligodendrocytes, or a decreased number of cellular processes.

To further assess the clinical applicability of our model, we measured cytokines that are putative mediators of these injuries in children with PVL and CP. We hypothesized that our model of intrauterine inflammation would cause an inflammatory response within the fetal brain, manifested by an increase in TNF- $\alpha$, IFN- $\gamma$, and IL-6. We hypothesized that the inflammatory response to our stimulus would be limited to Th1 cytokines and, therefore, the Th2 cytokine IL-10 would not be affected. Lastly, we hypothesized that the placenta would be a major source of cytokines released in response to the stimulus and, therefore, cytokines would be increased earlier in the placenta than the brain.

\section{MATERIALS AND METHODS}

All experiments were performed with the approval of the Institutional Animal Care and Use Committee of Children's
Research Institute of Children's National Medical Center. Timed-pregnant Fischer 344 and Lewis rats were obtained from Charles River Laboratories (Worchester, MA) at E12 and allowed to acclimate to our animal facility for $3 \mathrm{~d}$ before experimentation. LPS (Escherichia coli serotype 0111:B4) was obtained from Sigma Chemical Co. (St. Louis, MO). OptEIA ELISA kits for rat IFN- $\gamma$, TNF- $\alpha$, IL-6, and IL-10 were obtained from BD Pharmingen (San Diego, CA).

Experimental protocol. Pregnant rats were studied at E15 using a protocol we have previously described (35). Briefly, rats were anesthetized with $4 \%$ isoflurane, the cervix was visualized, and $0.1 \mathrm{mg} / \mathrm{kg}$ of LPS was administered in a mixture of $0.1 \mathrm{~mL}$ of saline. In pilot experiments to develop our model, we found that the cervical wall could be easily visualized using a surgical microscope and a small speculum. We found that we could reliably inject $0.1 \mathrm{~mL}$ of dye (Evans blue) within the cervical muscle with high reliability and without leakage into the surrounding tissue. Once injected with LPS, rats recovered from anesthesia and the procedure within 2 min of induction. We have previously established that this dose of LPS is associated with minimal fetal mortality (4\%) when animals are allowed to survive to delivery.

At designated times after injection (baseline, $1 \mathrm{~h}, 2 \mathrm{~h}, 6 \mathrm{~h}$, and $24 \mathrm{~h}$ ), rats were again anesthetized with $4 \%$ isoflurane. A laparotomy was performed and the uterus exposed. Fetal sacs were isolated, fetal brain and placenta were dissected and placed in $600 \mu \mathrm{L}$ of iced lysis buffer (RIPA buffer containing $50 \mathrm{mM}$ Tris- $\mathrm{HCl}, 1 \%$ NP-40, $0.25 \%$ Na-deoxycholate, 150 $\mathrm{mM} \mathrm{NaCl}$, and $1 \mathrm{mM}$ EDTA; recipe from Upstate, Charlottesville VA). Tissue was dissociated, sonicated, and centrifuged at $14,000 \mathrm{rpm}$ at $4^{\circ} \mathrm{C}$. Supernatants were collected and stored at $-70^{\circ} \mathrm{C}$ until analysis.

Protein determination. Protein concentration of each supernatant was determined using reagents from $\mathrm{BCA}$ following the directions from the manufacturer (Pierce Chemical, Rockford, IL). In brief, $2 \mu \mathrm{L}$ of supernatant was mixed with $48 \mu \mathrm{L}$ of deionized water and $1 \mathrm{~mL}$ of the BCA protein assay mixture. Standards were prepared similarly using BSA as a protein source. Samples were heated to $60^{\circ} \mathrm{C}$ in a water bath for $30 \mathrm{~min}$ and protein concentrations were determined using a spectrophotometer (Beckman DU Series 500, Beckman Coulter, Inc., Fullerton, CA) at $562 \mathrm{~nm}$.

Cytokine determination. TNF- $\alpha$, IL- $1 \beta$, IL-6, and IL-10 concentrations were determined by commercially available ELISA kits (BD Pharmingen) following the directions provided by the manufacturer. Samples $(100 \mu \mathrm{L})$ or known standard were added to wells coated with an appropriate capture antibody. After the wells were washed, a biotinylated antibody and an avidin-horseradish peroxidase conjugate were serially applied. Tetramethylbenzidine was used as a chromogen and the reaction was stopped after $30 \mathrm{~min}$ with the application of 1 $\mathrm{M} \mathrm{H}_{3} \mathrm{PO}_{4}$. Absorbance was measured at $450 \mathrm{~nm}$ by a microplate reader (Thermomax Microplate Reader, Molecular Devices, Sunnyvale, CA), and data were analyzed with Soft Max Pro software (Molecular Devices). Standard curves were determined from known standards and sample cytokine concentrations were calculated. Samples were run in duplicate, and the mean concentration of each sample per milligram of protein 
was calculated. For each cytokine, median concentrations of all the samples of a given time point were determined and reported as median $(25-75 \%)$.

Statistical analysis. Statistical analysis was performed using SPSS version 2.0 (SPSS Inc., Chicago, IL). Because we were interested in studying the acute effects of our LPS injection on baseline cytokine concentrations and not on saline controls, we compared concentrations at times after injection to baseline with the Kruskal-Wallis one-way ANOVA on ranks with Dunn's method. Correlations between cytokines were determined using Pearson correlations $(p<0.05$ was considered significant).

\section{RESULTS}

Four mothers per time period were studied (two Fischer 344 and two Lewis), producing a total of 80 placentas and fetal brains for analysis $(n=12,11,24,16$, and 17 at baseline, $1 \mathrm{~h}$, $2 \mathrm{~h}, 6 \mathrm{~h}$, and $24 \mathrm{~h}$, respectively). Preliminary analysis revealed no differences between Fischer 344 and Lewis rats for any cytokine at any time period studied in either the brain or placenta. This finding is consistent with our previous work demonstrating that each strain is equally affected by our inflammatory stimulus (35). Therefore, data from brains and placentas from both strains were combined for analysis at each time point.

Placental concentrations of the cytokines are summarized in Figure 1 . TNF- $\alpha$ significantly increased at $1 \mathrm{~h}$ and $2 \mathrm{~h}$ after the inflammatory stimulus, returned to baseline at $6 \mathrm{~h}$ and was
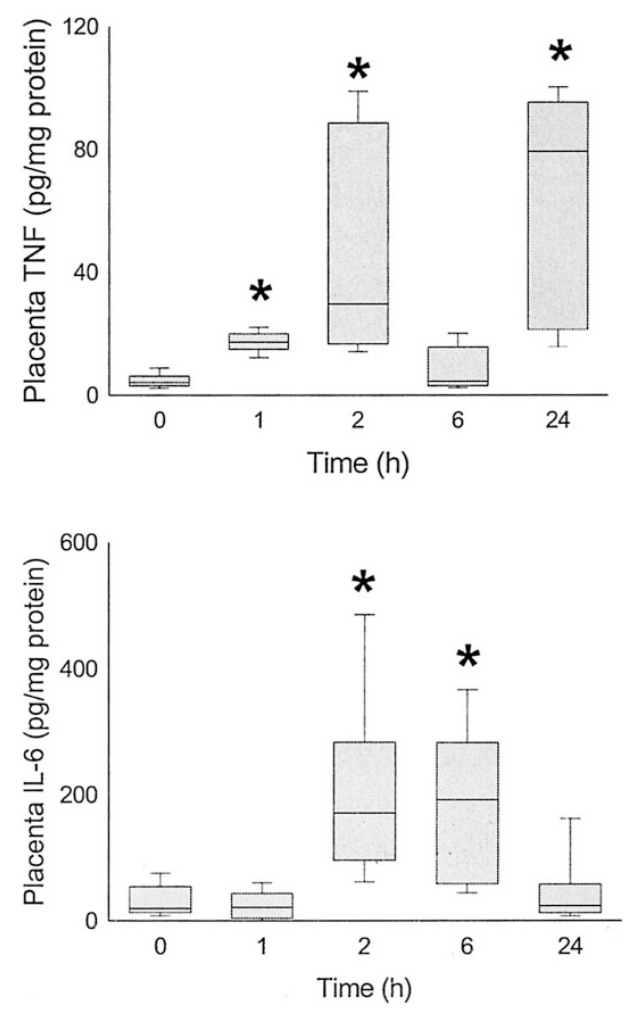

maximal (20-fold increase) at $24 \mathrm{~h}$ [baseline, $4.1 \mathrm{pg} / \mathrm{mg}(2.9-$ 5.7); 1 h, 17.2 (15.3-19.8); 2 h, 29.6 (16.6-88.4); 6 h, 4.5 (3.0-13.3); 24 h, 79.1 (22.5-94.5); $p<0.05$ for baseline versus $1 \mathrm{~h}, 2 \mathrm{~h}$, and $24 \mathrm{~h}$ ]. Conversely, placental concentrations of IFN- $\gamma$ remained unchanged during the initial time periods and decreased below baseline by 6 and $24 \mathrm{~h}$ [baseline, 13.6 $\mathrm{pg} / \mathrm{mg}$ (11.2-18.9); $1 \mathrm{~h}, 16.5$ (14.2-19.3); 2 h, 15.3 (7.9-26.7); 6 h, $6.1(0.9-8.0) ; 24$ h, $5.1(1.9-8.1) ; p<0.05$ for baseline versus $6 \mathrm{~h}$ and $24 \mathrm{~h}$ ]. Placental IL-6 concentrations were dramatically increased at $2 \mathrm{~h}$ and $6 \mathrm{~h}$ [baseline, $19.4 \mathrm{pg} / \mathrm{mg}$ (13.8-48.0); 1 h, 21.1 (13.8-41.4); 2 h, 170.6 (96.1-282.5); 6 h, 192.1 (61.1-269.4); 24 h, 23.2 (12.7-57.9); $p<0.05$ for baseline versus $2 \mathrm{~h}$ and $6 \mathrm{~h}$ ]. Placental IL-10 was mildly increased at $6 \mathrm{~h}$ and this increase persisted at $24 \mathrm{~h}$ [baseline, 2.5 $\mathrm{pg} / \mathrm{mg}(1.7-4.3) ; 1 \mathrm{~h}, 6.4$ (3.0-8.3); 2 h, 5.4 (1.6-9.2); $6 \mathrm{~h}$, 10.3 (5.8-13.2); 24 h, 6.0 (4.2-8.1); $p<0.05$ for baseline versus $6 \mathrm{~h}$ and $24 \mathrm{~h}]$.

Brain concentrations of cytokines are summarized in Figure 2. Brain TNF- $\alpha$ and IFN- $\gamma$ were both increased at all time periods after $2 \mathrm{~h}$ [TNF- $\alpha$ : baseline, $12.6 \mathrm{pg} / \mathrm{mg}(9.0-14.0) ; 1 \mathrm{~h}$, 11.9 (9.9-13.5); 2 h, $19.0(13.5-108.5) ; 6$ h, $111.9(88.1-$ 129.1); 24 h, 29.5 (19.5-34.8); IFN- $\gamma$ : baseline, $3.8 \mathrm{pg} / \mathrm{mg}$ (1.1-7.6); 1 h, 3.2 (1.6-6.8); 2 h, $38.4(8.8-72.4) ; 6$ h, 80.8 (65.4-91.7); 24 h, 20.3 (19.9-23.5); $p<0.05$ for baseline versus $2 \mathrm{~h}, 6 \mathrm{~h}$, and $24 \mathrm{~h}$ for both TNF- $\alpha$ and IFN- $\gamma$ ]. Brain concentrations of both IL- 6 and IL-10 showed slight declines during the study period, reaching significance below baseline at $24 \mathrm{~h}$ [IL-6: baseline, $142.3 \mathrm{pg} / \mathrm{mg}(110.7-185.3) ; 1 \mathrm{~h}, 164.8$
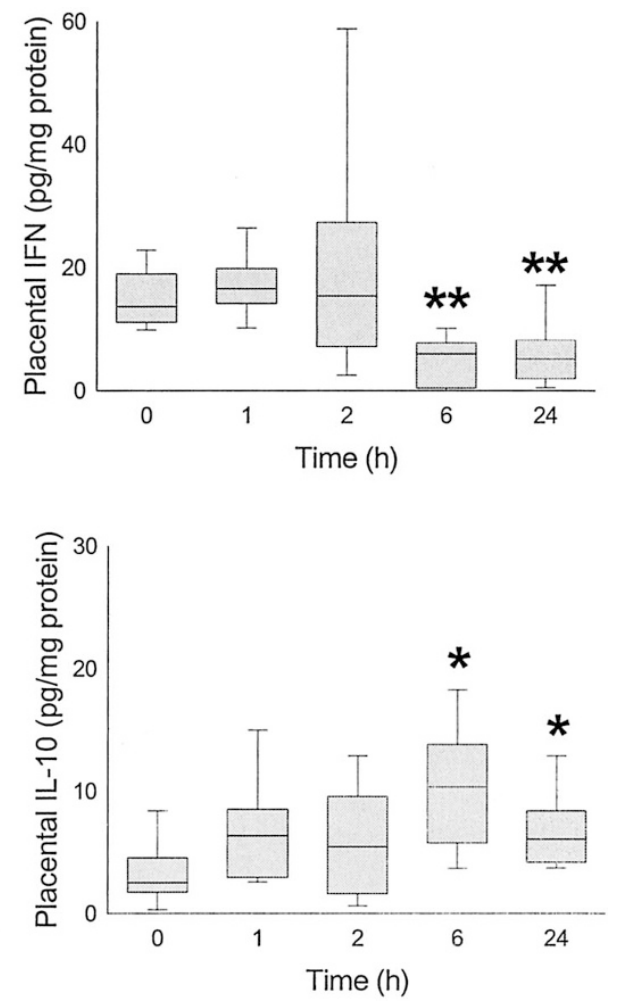

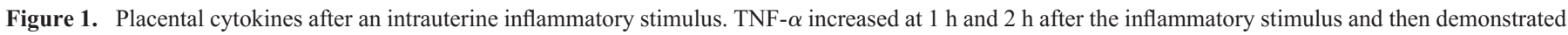

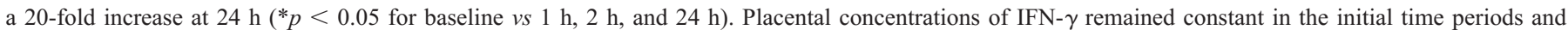

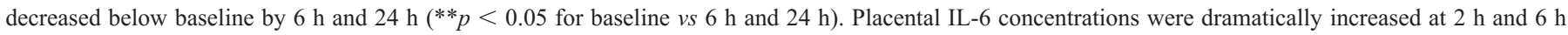
$\left({ }^{*} p<0.05\right.$ for baseline $v s 2 \mathrm{~h}$ and $\left.6 \mathrm{~h}\right)$. Placental IL-10 was mildly increased at $6 \mathrm{~h}$ and this persisted at $24 \mathrm{~h}(* p<0.05$ for baseline $v s 6 \mathrm{~h}$ and $24 \mathrm{~h})$. 

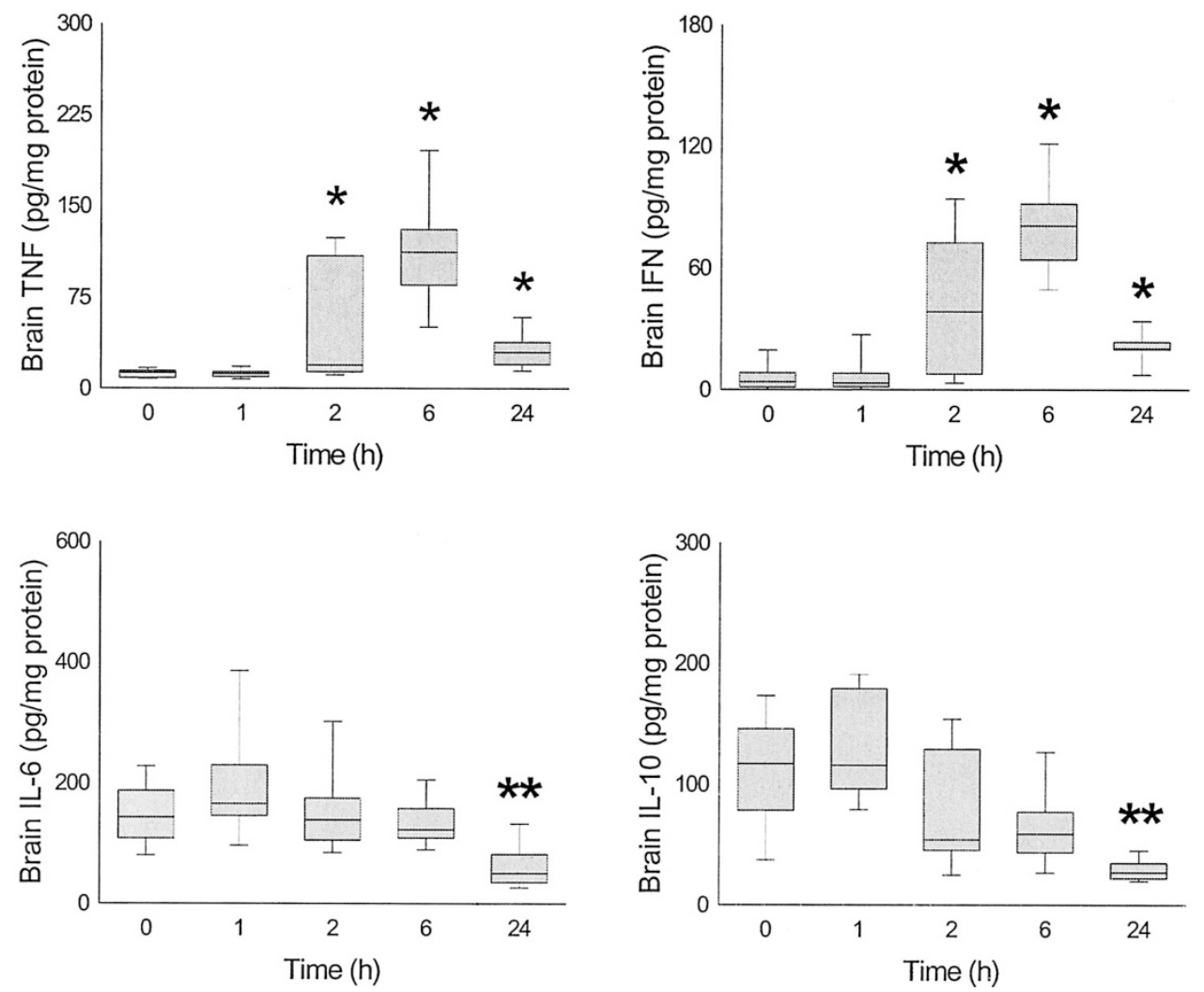

Figure 2. Brain cytokines after an intrauterine inflammatory stimulus. TNF- $\alpha$ and IFN- $\gamma$ were both increased at all time periods after $2 \mathrm{~h}\left({ }^{*} p<0.05\right.$ for baseline $v s 2 \mathrm{~h}, 6 \mathrm{~h}$, and $24 \mathrm{~h}$ for both TNF- $\alpha$ and IFN- $\gamma$ ). Brain concentrations of both IL- 6 and IL-10 showed slight declines during the study period, reaching significance below baseline at $24 \mathrm{~h}(* * p<0.05$ for baseline $v s 24 \mathrm{~h}$ for both IL-6 and IL-10).

(146.4-221.3); 2 h, $138.3(105.3-169.7) ; 6$ h, 122.2 (108.2155.9); 24 h, 49.7 (35.1-76.2); IL-10: baseline, $116.8 \mathrm{pg} / \mathrm{mg}$ (83.4-142.9); 1 h, $115.5(99.0-176.2) ; 2$ h, 54.0 (45.6-127.6); 6 h, 58.6 (45.7-75.3); 24 h, $26.6(21.8-33.7) ; p<0.05$ for baseline versus $24 \mathrm{~h}$ for both IL-6 and IL-10). In vitro studies suggest TNF- $\alpha$ and IFN- $\gamma$ are both required for toxicity of some glial cells, including oligodendrocytes and their precursors. We found a very high correlation between brain TNF- $\alpha$ and IFN- $\gamma\left(r^{2}=0.872, p<0.000001\right)$, suggesting both cytokines are increased concurrently.

\section{DISCUSSION}

In this study, we hypothesized that our inflammatory stimulus would increase Th1 cytokines, would not significantly change Th2 cytokines, and the placenta would be the main source of the cytokine response. Our results demonstrate a vigorous Th1 cytokine response in both the brain and placenta and minimal change in Th2 cytokine response in both the brain and placenta, thereby supporting the first two hypotheses. With regard to the final hypothesis, placental TNF- $\alpha$ was increased before an observed increase within the brain, suggesting that the placenta may be a fetal source of this cytokine. But, brain IFN- $\gamma$ increased dramatically without a placental IFN- $\gamma$ response, suggesting that the placenta is not a source of this cytokine in response to our stimulus.

In determining the utility of an animal model to study a human disease, we feel it is important that i) the cells and structures injured in the human condition are similarly affected in the animal model, ii) the putative mediators of injury in the human condition are present in the animal model, and iii) these mediators have a plausible rationale for producing the injury observed. Once these conditions are met, mechanisms of injury can then be assessed in the animal model and further comparisons can be made with the human disease.

With the completion of the present study, we believe we have begun to satisfy these criteria for our model. We have previously shown damage to the developing corpus callosum by oligodendrocyte-specific immunostaining (CNP and PLP) and an increased number of apoptotic nuclei after our inflammatory stimulus (35). In this study, we have found that two important Th1 cytokines are increased relatively early and persistently within the fetal brain after our stimulus is applied to the uterus of the mother. Others have found these cytokines are toxic to developing oligodendrocytes in culture $(28,29)$, so studying the mechanism of injury in our model may provide clues to the mechanisms of injury in children.

We are the first group to report on the placental contribution to the cytokine response and we have found that our intrauterine stimulus causes increased placental TNF- $\alpha$ and IL-6, but not IFN- $\gamma$. This suggests that although TNF- $\alpha$ produced in the placenta may simply cross the placental-fetal barrier, IFN- $\gamma$ is produced by the fetal immune system to cause its effects. In contrast, the IL-6 response observed within the placenta is not within the fetal brain. If this is proven true in other models and in humans, pharmacological treatments to mitigate these effects would necessarily need to target both the maternal and fetal immune systems. 
Models of inflammation have been developed in attempts to mimic developmental brain injuries. Several groups have instilled live bacteria within pregnant animals to develop an inflammatory response $(36,37)$ but quantification of the cytokine response in these models has not been reported. LPS administration has been used as an inflammatory stimulus in a variety of different methodologies. Duncan and colleagues (38) delivered LPS intravenously on 5 consecutive days. Serum IL-6 increased in the fetuses after the first two injections but then returned to baseline and other cytokines were not reported. This model also showed that LPS delivered in this manner had systemic effects on the developing fetus (decreased blood pressure and decreased oxygen saturation of arterial blood) and the treatment led to damage within the developing white matter. Our model did not estimate these physiologic changes caused by LPS injection due to size constraints in our animals. However, we measured tissue, rather than serum, concentrations of cytokines, which may be a more accurate reflection of the actions of these mediators. We chose to measure TNF- $\alpha$ and IFN- $\gamma$ due to their association with oligodendrocyte damage as well $(28,29)$. In the future, it would be interesting to determine the placental response to this intravenous method of LPS delivery.

Our findings are in relatively close agreement with models using intraperitoneal LPS injections. Cai and colleagues (39) injected timed-pregnant rats with LPS in two separate paradigms. In the first experiments, a relatively large dose of LPS $(4 \mathrm{mg} / \mathrm{kg})$ was administered on a single day (E18) and timedependent increases in TNF- $\alpha$ and IL- $1 \beta$ mRNA were observed in fetal brains. The mRNA of both cytokines were detectable at $1 \mathrm{~h}$ and maximal at $24 \mathrm{~h}$. In subsequent experiments, the authors injected LPS in a smaller dose $(500 \mu \mathrm{g} / \mathrm{kg})$ on both E18 and E19 and observed decreases in myelin basic protein staining within white matter structures at P8. Although this study reports mRNA concentrations rather than protein, the time course of TNF- $\alpha$ expression is similar to that observed in our study. Both studies confirm that LPS injection in pregnant animals can initiate a significant inflammatory response within the fetus, including the production of Th1 cytokines within the fetal brain. However, we observe this increase at the same dose of LPS that causes histologic changes later during development, and our model of injury assesses the placental cytokine response. Intraperitoneal injections of LPS bypass the placenta and may not mimic human chorioamnionitis as closely as models such as ours or those that involve bacterial infection of the uterus.

Others have directly injected LPS into the developing brain of developing animals to initiate the inflammatory response. Pang and colleagues (40) stereotactically injected LPS into the cerebral ventricles of P5 rats and found that the white matter was selectively damaged. At a number of time periods after the stimulus, brain TNF- $\alpha$, IL- 6 , and IL- $1 \beta$ concentrations were measured. They observed a vigorous TNF- $\alpha$ response within hours that persisted over $24 \mathrm{~h}$, which is similar to our findings. In contrast, they also observed a rapid increase in IL-6, which we did not observe. Recently, this group has shown that IL-1 $\beta$ may play a pivotal role in this model (41). We chose our cytokines because our preliminary data showed that TNF- $\alpha$ was present in the brain at the time of delivery whereas IL-1 $\beta$ was not observed. In the future, it would be interesting to determine whether our model shows a similar IL- $1 \beta$ response. Lenhardt and colleagues (42) injected LPS into the cerebral ventricles and similarly demonstrated selective injury to the developing white matter. Through a series of experiments, they deduced that LPS binding to TLR4 may be responsible for damaging the developing glia but they did not investigate the cytokine response in their experiments. Direct injection of LPS into the brain causes localized trauma and likely activates a wide variety of immunologic responses that may be additive or even synergistic in nature. Because epidemiologic studies as well as evidence from our model suggest that the placenta plays a role in this process, it is the burden of investigators using intracerebral injection models to prove that their stimulus mimics chorioamnionitis closely enough to make their applicability to humans apparent.

Our study has several limitations. We measured cytokine concentrations in the entire brain and this may not reflect the cytokine milieu within the true region of interest, the periventricular white matter. We considered dissecting the developing white matter from the adjacent structures but were concerned about introducing biases using this approach. Because developing white matter and gray matter have widely variable protein contents during development, inclusion of a small amount of adjoining structures might significantly affect the protein content and tissue homogeneity of the isolated samples. Because we standardized tissue cytokine concentrations to the total protein concentration of the homogenate, minor changes in the efficiency of the white matter dissection could cause significant bias in the results. Also, brain and placental tissue samples in this study included some amount of fetal blood. We strove to eliminate as much fetal blood contamination as possible, but the concentrations of cytokines we observed were necessarily influenced by some fetal blood contamination. This small amount of contamination probably explains why we observed minimal baseline concentrations of IFN- $\gamma$ in the brain. However, if the observed increases in cytokines were solely due to fetal blood, both placental and brain cytokine concentrations would have increased in parallel. As we demonstrated, IFN- $\gamma$ concentrations within the placenta did not increase whereas those in the brain increased 20 -fold. Lastly, we could not control for changes in protein synthesis caused by our LPS treatment. If the overall protein concentration of the fetal brains or placentas was changed by our treatment, then the cytokine concentrations that we calculated (standardized to overall protein content) would reflect this alteration. This effect would likely take some time to manifest itself and would be expected at the later time periods of the study. For instance, if our LPS treatment significantly increased overall protein synthesis, this would explain why brain IL-6 and IL-10 concentrations are decreased slightly at $24 \mathrm{~h}$ after treatment. Further studies to control for protein synthesis would be necessary to fully explain this effect.

In conclusion, we have demonstrated that our experimental model of intrauterine inflammation causes a vigorous and specific Th1 cytokine response in the fetal brain and placenta. Since these cytokines may play a role in the development of 
brain injuries in children, investigating the mechanisms of cell damage and death and the effects of our treatment on cells of the oligodendrocyte lineage may help in understanding these processes in children. If our findings of a fetal response to inflammation are proven true in other models and in children, pharmacological agents that can cross the placental barrier will be required for clinical trials to ameliorate the cytotoxic effects of inflammation. We believe that other studies seeking to mimic intrauterine inflammation should demonstrate that purported mediators in human disease are present at critical times during development.

\section{REFERENCES}

1. Wu Y, Colford J 2000 Chorioamnionitis as a risk factor for cerebral palsy: a meta-analysis. JAMA 284:1417-1424

2. Yoon BH, Jun JK, Romero R, Park KH, Gomez R, Choi JH, Kim IO 1997 Amniotic fluid inflammatory cytokines (interleukin-6, interleukin-1beta, and tumor necrosis factor-alpha), neonatal brain white matter lesions, and cerebral palsy. Am J Obstet Gynecol 177:19-26

3. Grether JK, Nelson KB 1997 Maternal infection and cerebral palsy in infants of normal birth weight. JAMA 278:207-211

4. Shalak L, Perlman J 2002 Infection markers and early signs of neonatal encephalopathy in the term infant. Ment Retard Dev Disabil Res Rev 8:14-19

5. Munoz-Fernandez M, Fresno M 1998 The role of tumor necrosis factor, interleukin 6 , interferon gamma and inducible nitric oxide synthase in the development and pathology of the nervous system. Prog Neurobiol 56:307-340

6. Gendron R, Nestel F, Lapp W, Baines M 1991 Expression of tumor necrosis factor alpha in the developing nervous system. Int J Neurosci 60:129-136

7. Arntzen KJ, Kjollesdal AM, Halgunset J, Vatten L, Austgulen R 1998 TNF, IL-1, IL-6, IL-8 and soluble TNF receptors in relation to chorioamnionitis and premature labor. J Perinat Med 26:17-26

8. Romero R, Mazor M, Sepulveda W, Avila C, Copeland D, Williams J 1992 Tumor necrosis factor in preterm and term labor. Am J Obstet Gynecol 166:1576-1587

9. Chung I, Benveniste E 1990 Tumor necrosis factor alpha production by astrocytes: induction by lipopolysaccharide, interferon gamma and interleukin 1 beta. J Immunol 144:2999-3007

10. Lee S, Liu W, Dickson D, Brosnan C, Berman J 1993 Cytokine production by human fetal microglia and astrocytes: differential induction by lipopolysaccharide and interleukin 1 beta. J Immunol 150:2659-2667

11. Farrar M, Schreiber R 1993 The molecular cell biology of interferon gamma and its receptor. Annu Rev Immunol 11:571-611

12. Boehm U, Klamp T, Groot M, Howard J 1997 Cellular responses to interferon gamma. Annu Rev Immunol 15:749-795

13. Ting J, Shigekawa B, Linthicum D, Weiner L, Frelinger J 1981 Expression and synthesis of murine immune response-associated (Ia) antigens by brain cells. Proc Natl Acad Sci U S A 78:3170-3174

14. Fierz W, Endler B, Reske K, Wekerle H, Fontana A 1985 Astrocytes as antigenpresenting cells I. Induction of Ia antigen expression on astrocytes by $\mathrm{T}$ cells via immune interferon and its effects on antigen. J Immunol 134:3785-3793

15. Turnley A, Miller J, Barlett P 1991 Regulation of MHC molecules on MBP positive oligodendrocytes in mice by IFN gamma and TNF alpha. Neurosci Lett 123:45-48

16. Neumann H, Boucraut J, Hahnel C, Misgeld T, Wekerle H 1996 Major histocompatibility complex (MHC) class I gene expression in single neurons of the centra nervous system: differential regulation by interferon gamma and tumor necrosis factor alpha. J Exp Med 185:305-316

17. Torres C, Aranguez I, Rubio N 1995 Expression of interferon gamma receptors on murine oligodendrocytes and its regulation by cytokines and mitogens. Immunology $86: 250-255$
18. Akira S, Taga T, Kishimoto T 1993 Interleukin-6 in biology and medicine. Adv Immunol 54:1-78

19. Vallieres L, Rivest S 1997 Regulation of the genes encoding interleukin-6, its receptor and gp130 in the rat brain in response to the immune activator lipopolysaccharide and the proinflammatory cytokine interleukin 1 beta. J Neurochem 69:1668-1683

20. Gadient R, Otten U 1994 Expression of interleukin-6 and interleukin-6 receptor mRNAs in rat brain during postnatal development. Brain Res 637:10-14

21. Gadient R, Otten U 1997 Interleukin-6-a molecule with both beneficial and destructive potentials. Prog Neurobiol 52:379-390

22. Vitkovic L, Maeda S, Sternberg E 2001 Anti-inflammatory cytokines: expression and action in the brain. Neuroimmunomodulation 9:295-312

23. Yoon B-H, Romero R, Tang SH, Jun JK, Kim I-O, Choi J-H, Syn HC 1996 Interleukin-6 concentrations in umbilical cord plasma are elevated in neonates with white matter lesions associated with periventricular leukomalacia. Am J Obstet Gynecol 174:1433-1440

24. Grether J, Nelson K, Dambrosia J, Phillips T 1999 Interferons and cerebral palsy. J Pediatr 134:324-332

25. Kashlan F, Smulian J, Shen-Schwarz S, Anwar M, Hiatt M, Hegyi T 2000 Umbilical vein interleukin 6 and tumor necrosis factor alpha plasma concentrations in the very preterm infant. Pediatr Infect Dis J 19:238-243

26. Zhang W, Wang L, Zhao Y, Kang J 2000 Changes in cytokine (IL-8, IL-6 and TNF-alpha) levels in the amniotic fluid and maternal serum in patients with premature rupture of the membranes. Zhonghua Yi Xue Za Zhi (Taipei) 63:311-315

27. Iida K, Takashima S, Ueda K 1995 Immunohistochemical study of myelination and oligodendrocytes in infants with periventricular leukomalacia. Pediatr Neurol 13:296-304

28. Cammer W 2000 Effects of TNF alpha on immature and mature oligodendrocytes and their progenitors in vitro. Brain Res 864:213-219

29. Selmaj K, Raine C, Farooq M, Norton W, Brosnan C 1991 Cytokine cytotoxicity against oligodendrocytes. J Immunol 147:1522-1529

30. Molina-Holgado E, Vela J, Arevalo-Martin A, Guaza C 2001 LPS/IFN-gamma cytotoxicity in oligodendroglial cells: role of nitric oxide and protection by the antiinflammatory cytokine IL-10. Eur J Neurosci 13:493-502

31. Trautman MS, Collmer D, Edwin SS, White W, Mitchell MD, Dudley DJ 1997 Expression of interleukin-10 in human gestational tissues. J Soc Gynecol Investig $4: 247-253$

32. Kline AE, Bolinger BD, Kochanek PM, Carlos TM, Yan HQ, Jenkins LW, Marion DW, Dixon CE 2002 Acute systemic administration of interleukin-10 suppresses the beneficial effects of moderate hypothermia following traumatic brain injury in rats. Brain Res 937:22-31

33. Spera PA, Ellison JA, Feuerstein GZ, Barone FC 1998 IL-10 reduces rat brain injury following focal stroke. Neurosci Lett 251:189-192

34. Dudley DJ, Hunter C, Mitchell MD, Varner MW 1997 Amniotic fluid interleukin-10 concentrations during pregnancy and with labor. J Reprod Immunol 33:147-156

35. Bell M, Hallenbeck J 2002 Effects of intrauterine inflammation on developing rat brain. J Neurosci Res 70:570-579

36. Yoon BH, Kim CJ, Romero R, Jun KJ, Park KH, Choi ST, Chi JG 1997 Experimentally induced intrauterine infection causes fetal brain white matter lesions in rabbits. Am J Obstet Gynecol 177:797-802

37. Debillon T, Gras-Leguen C, Verielle V, Winer N, Caillon J, Roze J, Gressens P 2000 Intrauterine infection induces programmed cell death in rabbit periventricular white matter. Pediatr Res 47:736-742

38. Duncan J, Cock M, Scheerlinck J-P, Westcott K, McLean C, Harding R, Rees S 2002 White matter injury after repeated endotoxin exposure in the preterm ovine fetus. Pediatr Res 52:941-949

39. Cai Z, Pan Z-L, Pang Y, Evans O, Rhodes P 2000 Cytokine induction in fetal rat brains and brain injury in neonatal rats after maternal lipopolysaccharide administration. Pediatr Res 47:64-72

40. Pang Y, Cai Z, Rhodes P 2003 Disturbance of oligodendrocyte development, hypomyelination and white matter injury in the neonatal rat brain after intracerebral injection of lipopolysaccharide. Brain Res Dev Brain Res 140:205-214

41. Cai Z, Pang Y, Lin S, Rhodes P 2003 Differential roles of tumor necrosis factor alpha and interleukin 1-beta in lipopolysaccharide-induced brain injury in the neonatal rat. Brain Res 975:37-47

42. Lenhardt S, Lachance C, Patrizi S, Lefebvre S, Follett P, Jensen F, Rosenberg P, Volpe J, Vartanian T 2002 The toll-like receptor TLR4 is necessary for lipopolysaccharide-induced oligodendrocyte injury in the CNS. J Neurosci 22:2478-2486 